www.jmscr.igmpublication.org

Impact Factor 5.84

Index Copernicus Value: 83.27

ISSN (e)-2347-176x ISSN (p) 2455-0450

crossref DOI: _https://dx.doi.org/10.18535/jmscr/v5i7.255

Journal Of Medical Science And Clinical Research

\title{
Stenotrophomonas Maltophilia- A Rare Cause of Prosthetic Valve Endocarditis: A Case Report and Review of Literature
}

\author{
Authors \\ Darshit Shah $^{1}$, Kunal Chawla ${ }^{2}$, Bhuwanesh Kandpal ${ }^{3}$ \\ ${ }^{1}$ Department of Internal Medicine, ${ }^{2}$ Department of Internal Medicine, ${ }^{3}$ Department of Cardiology \\ Sir Ganga Ram Institute of Postgraduate Medical Education and Research
}

\begin{abstract}
Background: Stenotrophomonas maltophilia (S. Maltophilia) is a gram-negative bacillus presumed to be a commensal and rarely causes opportunistic infections in immunocompromised individuals. Since it is resistant to most of the commonly prescribed antibiotics, it carries a high mortality. S. maltophilia infective endocarditis is extremely rare and only 43 cases have been reported worldwide till date.

Case Report: Our patient was a 45 years old male, known case of diabetes mellitus, Rheumatic heart disease (RHD) with previous aortic valve replacement, who had 2 episodes of S. maltophilia bacteremia and first episode being diagnosed as possible infective endocarditis being subsequently managed medically. However in the second episode of bacteremia diagnosis of infective endocarditis was confirmed and patient required prosthetic valve replacement.

Conclusion: This case is being presented to emphasize the possibility of infective endocarditis due to $S$. maltophilia and the clinicians should be aware of its potential significance in appropriate clinical setting and diagnosis should be considered early to prevent mortality.

Keywords: Stenotrophomonas Maltophilia, Infective Endocarditis.
\end{abstract}

\section{Introduction}

S. maltophilia is an aerobic, non-fermentative, gram-negative bacillus that is widely distributed in humans and the natural environment. It was presumed to cause opportunistic infections in humans rarely, except in those who were debilitated or were immunocompromised. ${ }^{1,2} \mathrm{~S}$. maltophilia infective endocarditis is a rare and poorly described disease. Although it is an opportunistic bacteria and less virulent than other causative organisms, it is difficult to treat as it is intrinsically resistant to various broad spectrum antibiotics. If untreated, it carries high morbidity and mortality rates. ${ }^{3}$ Hence it should be suspected as the cause of illness when isolated in at risk patients with early initiation of treatment.

We are reporting a rare case of S. maltophilia infective endocarditis involving prosthetic aortic valve with 2 episodes of bacteremia. Only 43 cases have been reported worldwide till date. ${ }^{4}$ To the best of our knowledge there are only two cases of S. maltophilia infective endocarditis reported from the Indian subcontinent so far and this is the $3^{\text {rd }}$ case overall. It is however the first case with two episodes of bacteremia reported from India.

\section{Case Report}

A 45 year old male, a known case of diabetes mellitus since 10 years and RHD since 35 years 
was apparently asymptomatic 15 days before presentation to the hospital, when he complained of high grade fever and breathlessness. He also gave history of burning micturition since 15 days. There was no history of predisposing events such as dental procedures or use of intravenous drugs. $\mathrm{He}$ had undergone closed mitral valvotomy (CMV) 30 years ago and aortic valve replacement 6 years ago for RHD and its sequelae. On initial examination he was found to be having a blood pressure of 100/70 $\mathrm{mm} \mathrm{Hg}$ and temperature $100 \mathrm{~F}$. General examination and examination of peripheral pulses was unremarkable and non contributory. On Cardiovascular examination, soft systolic murmur in mitral area was present and metallic click of prosthetic valve was audible in the aortic area. Respiratory, neurological and abdominal examination were unremarkable. His initial investigations revealed haemoglobin 11.6 gm per deciliter, total leukocyte count 9500 cells per cubic millimeter, platelets 198,000 cells per cubic millimeter and an Erythrocyte sedimentation rate of $45 \mathrm{~mm}$ at the end of the $1^{\text {st }}$ hour. His renal and hepatic parameters were within normal limits. Urine routine and microscopy was normal. Urine culture done on the $5^{\text {th }}$ day of fever showed growth of Escherichia Coli (Colony count $10^{5}$ colony forming units per millimeter). He was started on intravenous (IV) antibiotics which were given for a few days considering the initial diagnosis of urinary tract infection (UTI). On the $3^{\text {rd }}$ day of hospitalisation, blood culture showed growth of S. maltophilia. Initially it was presumed to be a commensal bacteria and IV antibiotics were continued as per the urine culture report. Echocardiography showed mild mitral stenosis and no evidence of endocarditis. As fever spikes persisted despite patient being on appropriate medication, alternative etiologies for fever were considered. Multiple blood cultures were sent from different sites, all of which grew $\mathrm{S}$. maltophilia. Transesophageal echocardiography (TEE) was done which did not reveal any vegetation. A diagnosis of possible infective endocarditis was made according to modified
Duke's criteria because of the absence of vegetations. According to the sensitivity reports, he was started on oral Co-trimoxazole and IV Minocycline and IV Ceftazidime which were given for a duration of 6 weeks. Patient became afebrile during the course of treatment and was subsequently discharged. Blood cultures done after 6 weeks of antibiotics were sterile. At home, patient remained afebrile and well for one week after stopping of the antibiotics when fever recurred. He was readmitted and repeat haemogram, renal and hepatic parameters were within normal limits. Repeat multiple blood cultures from different sites grew S. maltophilia. TEE was done again and this time it showed two soft echodense structures on the bioprosthetic aortic valve measuring $0.6 \mathrm{~cm}^{2}$ and $0.23 \mathrm{~cm}^{2}$. Hence the diagnosis of infective endocarditis was confirmed according to modified Duke's criteria in this $2^{\text {nd }}$ episode of bacteremia. He was again started on IV Ceftazidime, IV Minocycline and tablet Co-trimoxazole according to the sensitivity report. Patient was electively taken for aortic valve replacement and tissue specimen was sent for culture which grew S. maltophilia. Repeat blood culture after 6 weeks of antibiotics was sterile. On follow up he is afebrile and stable.

\section{Discussion}

S. maltophilia is an aerobic, non-fermentative, gram-negative bacillus measuring 0.5 to $1.5 \mu \mathrm{m}$ in length. ${ }^{1,2} \mathrm{~S}$. maltophilia infective endocarditis is rare. ${ }^{5,6}$ Risk factors for S. maltophilia infective endocarditis include IV drug abuse, use of central venous lines and history of prior cardiac surgery with prior valve replacement accounting for 40-60 $\%$ of the endocarditis cases. ${ }^{3,6,7,8}$ Carrillo-Cordova et al reported autoimmunity as a predisposing factor for S. maltophilia infective endocarditis. ${ }^{9}$ S. maltophilia is responsible for hospital acquired infections such as bacteremia, pneumonia, UTI, skin and soft tissue infections, ocular infections and meningitis. ${ }^{10}$ It is possible that the organism may be isolated when there is no clinical evidence of infection. ${ }^{11}$ True infection must be considered 
when it is isolated in a normally sterile specimen such as blood or CSF. ${ }^{12}$

S. maltophilia infections are difficult to treat because the organism is intrinsically resistant to many common broad-spectrum antibiotics and $\beta$ lactams. ${ }^{13}$ Sulphamethoxazole - trimethoprin is considered as the agent of choice. ${ }^{4}$ As it is a bacteriostatic drug, combination therapy is indicated to provide synergistic effect. ${ }^{4} \mathrm{~S}$. maltophilia infective endocarditis is associated with high mortality $(34.8 \%) .{ }^{4}$ Complications like cardiac failure, cerebral vascular accidents and organic abscesses are seen in $70-80 \%$ of patients. ${ }^{3,6,8}$

Prosthetic valves are involved in $51.1 \%$ of cases and surgery is required in $59.09 \%$ of patients having prosthetic valve $\mathrm{S}$. maltophilia infective endocarditis. Only $36.36 \%$ patients of prosthetic valves infective endocarditis are cured without surgery. ${ }^{4}$ The aortic valve was most frequently involved among prosthetic valves $(50 \%){ }^{4}$

Ours was a case of prosthetic aortic valve endocarditis with the possible risk factor being previous history of valve replacement. 6 weeks of usual antibiotic therapy didn't achieve a cure and the patient recovered only after the infected prosthetic valve was replaced followed by 6 weeks of effective multidrug antibiotic therapy.

\section{Conclusion}

As S. maltophilia infective endocarditis is rare and carries a high mortality due to its inherent resistance to commonly prescribed antibiotics, diagnosis should be considered early in patients having risk factors and treatment should be initiated as soon as possible. This is one of the extremely rare cases of S. Maltophilia infective endocarditis reported from india.

\section{References}

1. Palleroni NJ, Bradbury JF. Stenotrophomonas, a new bacterial genus for Xanthomonas maltophilia (Hugh 1980) Swings et al. 1983. Int J Syst Bacteriol. 1993;43:606-09.
2. Marshall WF, Keating MR, Anhalt JP, Steckelberg JM. Xanthomonas maltophilia: an emerging nosocomial pathogen. Mayo Clin Proc. 1989;64:1097-04.

3. Bayle S, Rovery C, Sbragia D, Brouqui P. Stenotrophomonas maltophilia prosthetic valve endocarditis: a case report. J Med Case Reports. 2008;2:174.

4. Suhani S, Patnaik A, Barik R, Nemani L. Infective endocarditis caused by Stenotrophomonas maltophilia: A report of two cases and review of literature. Indian Heart J. 2016;68:S267-S270.

5. Crum NF, Utz GC, Wallace MR. Stenotrophomonas maltophilia endocarditis. Scand J Infect Dis. 2002;34:925-27.

6. Meimoun P, Mainardi JL, Berrebi A, et al. Stenotrophomonas maltophilia endocarditis following mitral valve prosthesis implantation. Report of a case. Arch Mal Coeur Vaiss. 1999;92:1389-92.

7. Munter RG, Yinnon AM, Schlesinger Y, Hershko C. Infective endocarditis due to Stenotrophomonas (Xanthomonas) maltophilia. Eur J Clin Microbiol Infect Dis. 1998; 17:353-56.

8. Khan IA, Mehta NJ. Stenotrophomonas maltophilia endocarditis: a systematic review. Angiology. 2002;53:49-55.

9. Carrillo-Córdova JR, Amezcua-Guerra LM. Autoimmunity as a possible predisposing factor for Stenotrophomonas maltophilia endocarditis. Arch Cardiol Mex. 2012;82:204-07.

10. Sattler CA, Mason EO Jr, Kaplan SL. Nonrespiratory Stenotrophomonas maltophilia infection at a children's hospital. Clin Infect Dis. 2000;31:1321-30.

11. Aoun M, Van der Auwera P, Devleeshouwer $\mathrm{C}$, et al. Bacteraemia caused by non-aeruginosa Pseudomonas species in a cancer centre. J Hosp Infect. 1992;22:307-16.

12. Kim JH, Kim SW, Kang HR, et al. Two episodes of Stenotrophomonas maltophilia 
endocarditis of prosthetic mitral valve: report of a case and review of the literature. J Korean Med Sci. 2002;17:26365.

13. Vartivarian S, Anaissie E, Bodey G, Sprigg H, Rolston K. A changing pattern of susceptibility of Xanthomonas maltophilia to antimicrobial agents: implication for therapy. Antimicrob Agents Chemother. 1994;38:624-27. 